\title{
Exacerbation of Streptococcus Equi (Strangles) by Overly Nutritious Diets in Horses: A Model for Infectious Bacterial Diseases of Horses and Other Livestock
}

\author{
Thomas Walter Swerczek \\ Department of Veterinary Science, 108 Gluck Equine Research Center, University of Kentucky, Lexington, USA
}

Email address:

twswer1@uky.edu

To cite this article:

Thomas Walter Swerczek. Exacerbation of Streptococcus Equi (Strangles) by Overly Nutritious Diets in Horses: A Model for Infectious Bacterial Diseases of Horses and Other Livestock. Animal and Veterinary Sciences. Vol. 7, No. 1, 2019, pp. 18-23.

doi: $10.11648 /$ j.avs.20190701.13

Received: February 1, 2019; Accepted: March 8, 2019; Published: March 29, 2019

\begin{abstract}
Strangles is a highly pathogenic and contagious infection of horses and other equids caused by Streptococcus equi. Reports in the literature summarize the severe complications of strangles, but provide little information on the factors enhancing the pathogenicity and complications that result in high mortalities. However, there are reports suggesting exceptionally nutritious diets will exacerbate strangles infections and promote complications, including bacteremia and metastases to lymph nodes throughout the body and sequalae like purpura hemorrhagic and myositis. Dietary ingredients may enhance S. equi capsular polysaccharide (CPS) which protects bacteria from the immune system and phagocytosis. Lowvirulent bacteria may become highly virulent pathogenic bacteria. Overly nutritious diets enhance the pathogenicity of S. equi and efficiency of bacterins, vaccines and antibiotics intended for controlling and treating strangles in horses. The effect of diet on the pathogenesis of S. equi in horses may be an ideal model for other bacterial diseases of horses as well as bacterial diseases like Pasturella multocida respiratory infections in cattle and other livestock.
\end{abstract}

Keywords: Strangles, Streptococcus Equi, Complications, Sequalae, Overly Nutritious Diets

\section{Introduction}

Strangles is a highly pathogenic and contagious infection of horses and other equids caused by Streptococcus equi [1]. The acute bacterial disease is an upper respiratory infection characterized by rhinopharyngitis, serous and mucopurulent ocular and nasal discharge, pyrexia, coughing, and suppurative lymphadenitis of the submandibular, submaxillary and retropharyngeal lymph nodes [2-3]. Strangles may become exacerbated and chronic if the disease is interfered by detrimental factors. Complications that may occur are intensified suppuration in the retropharyngeal lymph nodes, causing obstruction of respiration, rupture of the lymph nodes into the guttural pouches causing an empyema. Severe strangles infections can be fatal because of a variety of complications that occur [2-3]. Bacteremia may occur with metastases to the periorbital, bronchial, mesenteric and other lymph nodes forming chronic abscesses. S. equi may also infect the brain with fatal neurologic lesions [4]. Complications of S. equi infections are often referred to as bastard strangles (metastatic abscessation). Purpura hemorrhagica, myositis, and other syndromes are sequalae to strangles complications [3].

Strangles is associated with high morbidity and low mortality with a duration of 25 to 35 days. However, factors inducing exacerbation and complications may extend the infection to become chronic and endemic. In exacerbated strangles, the enlarged and abscessed retropharyngeal lymph nodes may rupture into the guttural pouches causing an empyema resulting in chronic infections and a source for constant shedding of $\mathrm{S}$. equi through nasal secretions. Chronic carrier horses with empyema of the guttural pouches may be a source of $\mathrm{S}$. equi in endemic herds for months and years [5-6].

\section{Review of Literature on Strangles Complications}

There are several reports in the literature on strangles complications and sequalae, but causative factors responsible 
for S. equi exacerbations have yet to be established. However, one report suggests that the key risk factor is overly nutritious diets [7]. In another report by Ford and Lokai, they describe strangles complications and sequelae that were fatal in weanling horses consuming a concentrated nutritious diet [8]. Their description of the diet fed to a herd of horses with multiple fatal cases of strangles with complications and sequalae was as follows: "All these weanlings were fed more or less like cattle under feedlot conditions. Any roughage that was fed was of extremely poor quality so much so that many of the horses would not even eat it. Many weanlings could ignore oats and eat many pounds of calf manna (Carnation)." (Calf manna supplements, like alfalfa, contain multiple abundant sources of high-quality proteins, carbohydrates, vitamins and minerals for use as a supplement, not as a total feed source). Their description of the diet fed to a herd of horses with fatal strangles complications was consistent with a concentrated overly nutritious diet. In other reports on strangles complications and sequalae, the dietary ingredients including supplements fed were not discussed or recorded.

Necropsy examinations on 85 horses with strangles complications and sequalae that were fatal, and the epidemiologic findings on endemic farms with strangles revealed that there was an association between diet and strangles complications [7]. These fatal cases were from the central Kentucky area where spring pastures were high in protein and often laden with white clover. In addition, affected horses were being supplemented with a variety of concentrated protein, carbohydrate, mineral and vitamin supplements, and alfalfa. In these herds, there were inconsistencies as to the effectiveness of antibiotics, bacterins and vaccines in treating and preventing $\mathrm{S}$. equi infections. Therefore, the effects of diets on the pathogenesis and the efficacy of antibiotics for S. equi infections in horses were performed in controlled experimental trials [7].

\subsection{Effects of Diet and Antibiotics on Strangles Complications in Experimentally S. equi Infected Horses}

In separate experimental trials, 133 yearling horses of mixed light horse breeds from a strangle-free herd were infected intranasally with a pathogenic strain of S. equi. All infected horses were maintained on a low plane of nutrition. The ration was oats and mixed grass-legume hay consisting of timothy hay and orchard grass and a limited amount of red clover. White salt was fed free choice. No additional supplementation was provided. Antibiotics tested included penicillin, ampicillin, sulfa drugs, and trimethoprin/sulfadiazine. Control and treated horses were necropsied at 4 to 5 weeks post infection.

\subsection{Results of Diet and Antibiotics on Strangles Complications in S. equi Infected Horses}

These antibiotics were found to be effective for preventing S. equi infections if used before the first day of pyrexia, and before any lymph node enlargement or abcessation occurred. After the lymph nodes became abscessed, the infections were prolonged as long as the antibiotics were given. No complications or bastard strangles, other than prolonged $\mathrm{S}$. equi infections treated with antibiotics, occurred in any of the 133 control or antibiotic-treated infected horse. All infections were restricted to the regional upper respiratory lymph nodes, and the guttural pouches were not affected with empyema. However, another trial of 12 light-horse type, mixed-breed yearling horses, a potent antibiotic not approved for use in horses induced severe bastard strangles in all antibiotictreated horses, but not in any of the untreated infected controls on the same diet of mixed grass-legume hay and oats.

These results demonstrated that $\mathrm{S}$. equi infections without complications affect primarily the submandibular and retropharyngeal lymph nodes with lymphadenitis and abscessation. In the 133 experimental horses that were not fed an overly nutritious diet, no strangles complications or bastard strangles occurred. By contrast, 85 Thoroughbred, Arabian, Standardbred, Quarter horses, and ponies with strangles complications that were examined at necropsy due to fatal strangles infections had a history of being fed overly nutritious diets. Metastatic abscessation of S. equi occurred in lymph nodes and organs throughout the body, including the brain. Epidemiologic findings on several horse farms with endemic strangles had a history of over nutrition [7] similar to that recorded by Ford and Lokai [8].

\subsection{Interrelationship Between Diet and Infectious Diseases with Strangles as a Model}

There is an interrelationship between the type of diets and infectious and metabolic disease syndromes of horses and other livestock. Using S. equi as a model to demonstrate this phenomenon a controlled experimental trial was performed [7].

Six weanling light horse mixed breed foals from a strangles-free herd were randomly divided into two groups of three each. Group 1 was fed a high-protein alfalfa hay obtained from a Standardbred horse farm with the same type of hay fed to horses with endemic strangles year after year with severe complications and other sequalae related to strangles infections. The alfalfa hay was dehydrated in the form of large cubes. S. equi was isolated from an infected horse on the same Standardbred farm where high-protein alfalfa was being fed and had history of endemic and strangles complications for years. Group 2 was fed timothy hay and oats. Both groups had access to free-choice white salt. The 6 weaning foals were housed in the same isolation facility and two randomly selected groups of three were separated by a portable gate so the only variable was the type of diet being fed to each group.

$\mathrm{S}$. equi was cultured in Todd-Hewitt broth. Ten cc of a 24hour log phase culture was inoculated intranasally into all foals in both groups. Three foals in group 1 that were fed high-protein alfalfa hay developed severe clinical signs of strangles with pyrexias up to 107 degrees $\mathrm{F}$, mucopurulent 
ocular and nasal discharge, marked lymphadenitis affecting the submandibular and retropharyngeal lymph nodes. One foal developed severe respiratory signs of pneumonia and was euthanized at two weeks post-infection because of impending death. At necropsy, the lymph nodes were very enlarged, especially the retropharyngeal lymph nodes. There were metastases with abscesses in the bronchial lymph nodes and lungs. At three weeks post-infection the two remaining foals were necropsied. The submandibular, submaxillary and retropharyngeal lymph nodes were very enlarged and abscessed, especially the retropharyngeal lymph nodes. All abscessed lymph nodes were positive for $\mathrm{S}$. equi.

Two foals fed timothy hay and oats in group 2 developed only mild clinical signs of pyrexia of 103 degrees F. at 48 hours after the start of pyrexia in group 1. There was no evidence of mucopurulent ocular, nasal discharge, or regional lymphadenitis. Necropsy, at 3 weeks post-infection, only the retropharyngeal lymph nodes of both foals were mildly enlarged with very little abscessation and both were positive for $\mathrm{S}$. equi. The third foal in group 2 did not develop pyrexia or any clinical signs of strangles. Upon necropsy at three weeks post-infection, there was no evidence of any lymphadenitis or lymph node abscessation and the regional lymph nodes were negative for S. equi. If foals in group 2 which were on a low plane of nutrition were not necropsied, S. equi infection would not have been detected as there were no typical clinical signs of strangles.

The results of the controlled experiment demonstrated that there was a dramatic difference in the virulence and pathogenicity of the S. equi infections in foals fed highprotein alfalfa hay compared to the low plane of nutrition of timothy hay and oats.

\subsection{Effects of Dietary Ingredients on the Pathogenicity of Microorganisms with S. equi as a Model}

Dietary ingredients from autoclaved water extracts of the timothy and alfalfa hay that were fed to foals in the experimental trial in section 2.3 were inoculated with the same $\mathrm{S}$. equi isolate used in the experimental trial. The bacterial growth in the timothy hay extract was sparse with bacteria in short chains that lacked any capsular material. By contrast, the bacterial growth in the alfalfa extract was very abundant with long chains of bacteria with robust capsular material. These in vitro tests on the dietary extracts demonstrated that there was abundant bacterial growth containing marked capsular material when $\mathrm{S}$. equi was grown in an extract of alfalfa hay as compared to that grown in timothy hay extract.

\section{Discussion}

These findings offer evidence that dietary ingredients play a central role in the pathogenesis of bacterial infections of $\mathrm{S}$. equi in horses and likely for infectious bacterial diseases of other livestock. This study demonstrated that dietary ingredients affect the production of capsular polysaccharide (CPS) by pathogenic bacteria.
There is an association between (CPS) and the pathogenicity of S. equi [9]. Mucoid polysaccharide (hyaluronic acid) influences the virulence but it may depend on the presence or absence of other factors, like M-protein [10]. An essential virulence factor of Pasturella multocida is a glycosaminoglycan capsular polysaccharide (CPS) that helps protect surface antigens from the host's immune system and prevents phagocytosis and bactericidal activity and interferes with Biofilm formation [11]. Studies with S. equi as a model offer evidence that low-virulence strains of bacteria may revert to highly virulent strains if animals are exposed to overly nutritious diets. For maximum production of CPS, essential nutrients are necessary in artificial media. It is apparent that essential nutrients in dietary ingredients fed to horses with $\mathrm{S}$. equi infections are also necessary for maximum production of CPS. Seemingly, overly nutritious high-protein diets enhance the virulence and pathogenicity of S. equi to become super bacteria due to robust CPS and possibly other factors.

There are apparently strains of S. equi with different levels of virulence, but all strains are seemingly pathogenic and not part of the normal flora. Unlike S. equi, Pasturella multocida, may be part of the normal flora of the respiratory tract of livestock like swine, cattle and other animals [11] and if animals are on a low plane of nutrition, these bacteria may be innocuous. However, if the plane of nutrition is acutely elevated, Pasturella spp. and other bacterial species may become super bacterial infections, like $\mathrm{S}$. equi complications, and may be fatal. This typically occurs in weanling calves after being acutely exposed to overly nutritious diets under feed-lot conditions [12]. Over nutrition not only affects the host but their microbiome. When there are pathogens within the normal flora, exceptionally nutritious diets stimulate the overgrowth of pathogens and increase their virulence and pathogenicity to the extent that it may overwhelm the immune system and protective factors from beneficial normal flora.

It was observed that spring pastures high in protein, especially those laden with legumes also exacerbated strangles infections in horses. Horses have a higher incidence of strangles complications in the spring than in summer months [13]. Pastures are more nutritious in the spring due to higher protein than during summer months. It was found that ponies, light-horse types, Quarter horses, Standardbred horses, Arabian, and Draft horses were more susceptible to S. equi infections than Thoroughbred horses. Seemingly, a higher plane of nutrition is required for Thoroughbreds than other breeds. However, it was observed when Thoroughbred horses were fed overly nutritious diets, especially while grazing high protein spring pastures and being supplemented with protein-grain rations and high-protein alfalfa hay, they were as susceptible as other breeds and bastard strangles was common [7]. For meaningful comparative results between different experimental trials or reporting of strangles on affected farms with complications, it is essential to record age, breed, sex, strain, and dietary ingredients fed to experimental horses and horses with spontaneous strangles. 
These singalments and type of diet dramatically affect the pathogenesis and complications of strangles in horses.

Persistent strangles in horses is difficult to eliminate if the plane of nutrition is high. Strangles may be present for months or years, especially on farms with new foals each year. To eradicate strangles from a herd the plane of nutrition must be lowered if affected horses are being overfed. If horses are grazing high-protein legume pastures, moving them to a dry lot or less nutritious pastures until they are negative for S. equi may be necessary. Chronically infected strangles horses commonly harbor S. equi in their guttural pouches and excrete it through their nasal secretions. No new horses should be added to the herd until all horses are negative for $\mathrm{S}$. equi by culture and/or molecular methods. In chronic strangles with lymph node abscesses, antibiotics may be problematic as they may prolong infections. The infected abscesses should be allowed to clear and become negative while being fed a safe dietary ration.

Overly nutritious diets may exacerbate and induce super infections, and the overgrowth of S. equi which overwhelms the immune system and the efficacy of bacterins, vaccines and antibiotics. However, when horses are exposed to a low plane of nutrition, bacterins, vaccines, and approved antibiotics appear to be somewhat efficacious. Vaccines based on $\mathrm{M}$ protein or inactivated bacterial suspensions may reduce the clinical attack rate by $50 \%$, a level of protection much lower than that produced during recovery from strangles [14]. Antibiotics, bacterins, and vaccines for the treatment and prevention of strangles have been a contentious issue because the type of diet fed has not been considered [15-19].

Due to the type of gastrointestinal tract and microbiome in their gut, horses can assimilate a high-fiber diet. However, they do have a tendency to overeat a nutritious diet more so than other animals if not limited or controlled. High carbohydrate and protein rations may be problematic for a variety of disease syndromes. In addition to strangles, feeding of overly nutritious high-protein diets to lactating mares predispose their foals to neonatal Clostridial diseases [20-21]. In addition, other bacterial diseases, including Corynebacterium equi and Lawsonia intracellularis were also observed to be associated with overly nutritious diets fed to lactating mares or weaning foals.

It was found that alfalfa exacerbates strangles and neonatal diseases in foals more than other high-protein sources. Alfalfa has abundant proteins, amino acids, vitamins, minerals, and trace elements. In addition to high-protein levels, and other nutrients, alfalfa is an accumulator of nitrate, and a source of sulfur, which may be contributing to the exacerbation of infectious diseases [22].

Dietary ingredients affect the animal per se and the microbiome of a variety of microorganisms in the animal's respiratory and gastrointestinal tracts. These commensal and symbiotic microorganisms benefit their host and provide essential nutrients and inhibitory factors against pathogens. However, when the microbiome contains opportunistic pathogens, highly nutritious ingredients may result in unintended consequences by promoting multiplication and pathogenicity of pathogens like S. equi. For this reason, a lower plane of nutrition is desirable and beneficial in allowing the normal microbiome to be maintained and to compete with pathogens by inhibiting their multiplication. Similarly, antibiotics may eliminate beneficial microflora and allow pathogens to dominate and exacerbate diseases of horses like strangles and other infectious diseases [23-24]. Rather than using antibiotics to prevent and treat super bacterial infections in livestock, the risk factors like overly nutritious diets and causative nutrients should first be addressed. Then, bacterins and vaccines may be more efficacious in preventing diseases, and antibiotics would not be necessary.

High-protein diets can be eliminated or lessened in controlled single feeding arrangements, but when horses are fed in groups as described by Ford and Lokai [8], some horses may overeat and be affected with complications of strangles. When horses are fed individually, the dietary intake can be managed and controlled. However, it is difficult to control overeating when horses are grazing high protein, clover-laden spring pastures. This is why strangles complications are common during the spring when pastures are high in protein and may be overly nutritious as compared to summer pastures that are lower in protein and when complications of strangles are less common.

Empyema of the guttural pouches is a common source of shedding in chronic and endemic S. equi infections. None of the 133 experimentally infected horses fed a low plane of nutrition developed guttural pouch empyema [8]. Empyema of the guttural pouches was induced in 9 of 20 horses with experimentally induced strangles. However, the type of diet fed to these horses was not recorded [6].

Strangles is a serious disease in horses that affects their movement for a variety of events and affects the equine industry worldwide. Attempts should be made to develop methods to eradicate S. equi infections in horses [25-28]. In addition, culture techniques, molecular methods, including PCR and ELISA diagnostic tests may be used to replace culture methodologies to detect carrier infected animals. Biosecurity, screening procedures and improved bacterins and vaccines are critical in controlling the spread of S. equi infections [29]. However, unless the role of nutrition is dealt with, the exacerbation and pathogenicity of S. equi infections will be problematic, and the control and eradication of strangles infections in horses will remain a formidable task.

\section{Conclusions}

Strangles has a high morbidity and low mortality with a duration of 25 to 35 days if horses are not fed an overly nutritious diet. Some horses may not display any clinical signs of strangles, yet be infected with $\mathrm{S}$. equi, on a low plane of nutrition. However, overly nutritious diets may exacerbate $\mathrm{S}$. equi infections and induce complications. These include metastases of $\mathrm{S}$. equi to lymph nodes and organs throughout the body and other sequalae like purpura 
hemorrhagica and myositis.

In exacerbated strangles the enlarged and abscessed retropharyngeal lymph nodes may rupture into the guttural pouches causing an empyema, which results in chronic infections and a source for constant shedding of $\mathrm{S}$. equi through nasal secretions. These chronic carrier horses with empyema of the guttural pouches are a source of $\mathrm{S}$. equi in endemic herds for months and years especially on farms with additional horses or newborn foals each year.

To eliminate strangles from a group of horses it is suggested to allow the disease to come to a natural conclusion. New horses should not be added to a herd with strangles until all infected horses are negative for $\mathrm{S}$. equi while eliminating risk factors that promote super infections. Primarily among these are antibiotics and exceptionally nutritious diets that may enhance the multiplication, virulence and pathogenicity of $\mathrm{S}$. equi. Antibiotics are ineffective after S. equi abscesses develop in lymph nodes and other organs. Antibiotics may disrupt the beneficial microbiome of the upper respiratory tract that provide inhibitor factors against pathogens like S. equi. The efficacy of bacterins and vaccines may vary depending on the plane of nutrition and nutrients fed to affected horses. The level of protection from vaccination is much lower than that produced during recovery from strangles, and excessive nutrition may overwhelm any immunity and strangles can reoccur.

Biosecurity, quarantine, improved culturing and molecular testing procedures are critical in controlling the spread of S. equi infections. However, exacerbation factors, including overly nutritious diets need to be addressed before endemic strangles can be eliminated. Overly nutritious dietary ingredients enhance the production of CPS which protects bacteria from the immune system and phagocytosis allowing low-virulent bacteria to become highly virulent pathogenic bacteria like S. equi in horses [7]. S. equi infections or strangles in horses may be a model for other infectious bacterial diseases of horses, and diseases in other livestock, like Pasturella multocida [11] in feedlot cattle [12] that are acutely fed overly nutritious diets.

\section{Conflicts of Interest}

The author declares that there are no conflicts of interest.

\section{References}

[1] Bryans, J. T., Doll, E. R, Shephard, B. P. (1964) The etiology of strangles. Cornell Vet. 54, 198-205.

[2] Sweeney, C. R., Whitlock, R. H., Meirs, D. A., Whitehead, S. C, Barningham, S. O. (1998) Complications associated with Streptococcus equi in a horse farm. JAVMA. 191, 1446-1448.

[3] Boyle, A. G. (2017) Strangles and its complications. Equine Vet. Educ. 29(3), 149-157. doi: 10.1111/eve.12568

[4] Spoormakers, T. J., Ensink, J. M., Goehring, L. S., Koeman, J. P., Ter Braake, F., van der Vlugt-Meijer, R. H., van den Belt, A. J. (2003) Brain abscesses as a metastatic manifestation of strangles: symptomatology and the use of magnetic resonance imaging as a diagnostic aid. Equine Vet J. 35(2), 146-151.

[5] Newton J. R., Wood J. L. N., Dunn K. A., DeBrauwere M. N., Chanter N. (1997) Naturally occurring persistent and symptomatic infection of the guttural pouches of horses with Streptococcus equi. Vet. Rec. 140, 84-90.

[6] Knight A. P., Voss J. L., McChesney A. E., Bigbee H. G. (1975) Experimentally-induced Streptococcus equi infection in horses with resultant guttural pouch empyema. Vet Med Small Anim Clin. 70(10), 1194-1199.

[7] Swerczek, TW. Pathogenesis, Treatment and Control of Streptococcus equi Infection (strangles) in Horses. Proceeding of the First Annual Eastern States Veterinary Conference, Orlando. Fl. January 18, 1984.

[8] Ford, J., Lokai M. D. (1980) Complications of Streptococcus equi infections. Equine Pract. 2, 41-44.

[9] Bin Xu, Xiaomeng Pei, Yiqi Su, Zhe Ma, Hongjie Fan. (2016) Capsule of Streptococcus equi subsp. zooepidemicus hampers the adherence and invasion of epithelial and endothelial cells and is attenuated during internalization. FEMS Microbiology Letters, 363 (16) https: doi.org/10.1093/femsle/fnw164.

[10] Woolcock, J. B. (1974) The capsule of Streptococcus equi. J. of General Microbiology. 85, 372-375.

[11] Petruzzi, B., Briggs, R. E., Tatum, F. M., Swords, W. E., De Castro, C., Molinaro, A., Inzana, T. J. (2017) "Capsular Polysaccharide Interferes with Biofilm Formation by Pasteurella multocida Serogroup A"J American Society of microbiology. Volume 8, no. 6. doi:org/10.1128/mBio.01843-17

[12] Swerczek, T. W. The Interrelationship between Nutrition and Infectious Diseases of Livestock. East Central Nebraska Cattlemen Association Meeting. November 20, 1998. Boone County, Nebraska.

[13] Duffee, L. R., Stefanovski, D., Boston, R. C., Boyle, A., G. (2015) Predictor variables for and complications associated with Streptococcus equi subsp equi infection in horses. JAVMA. 247:1161-1168. Doi:10.2460/javma.247.10.1161.

[14] Timoney, J. F. (1993) Strangles. Vet. Clin. N. Am. Equine Pract. 9, 365-374.

[15] Bazeley, P. L. (1942) Studies with Equine Streptococci, 3. Vaccination against strangles. Austral. Vet. J. 18, 141-155.

[16] Woolcock J. B. (1975) Immunity to Stretococcus equi. Aust Vet J. 51, 554-559.

[17] Waller A. S., Jolley K. A. (2007) Getting a grip on strangles: recent progress towards improved diagnostics and vaccines. Vet J. 173, 492-501.

[18] Timoney, J. F. (2007) Strangles vaccines in trouble again. Equine Vet. J. 39, 196. doi.org/10.2746/042516407X196744

[19] Fairley, J. K. (1991) Strangles in horse studs: incidence, risk factors and effect of vaccination. Aust Vet J. 68(8), 282-283.

[20] Swerczek, T. W. (2013) Tyzzer's Disease in Foals: Retrospective studies from 1969 to 2010. Canadian Veterinary Journal, 54, 876-880.

[21] Swerczek, T. W. (1980) Toxicoinfectious Botulism in Foals and Adult Horses. JAVMA 176, 217-220. 
[22] Swerczek, T. W., Dorton, A. R. (2019) Effects of Nitrate and Pathogenic Nanoparticles on Reproductive Losses, Congenital Hypothyroidism and Musculoskeletal Abnormalities in Foals: New Hypotheses. Animal and Veterinary Sciences. 7 (1), 1-11 doi: $10.11648 /$ j.avs.20190701.11

[23] Swerczek, T. W. (1978) Inhibition of the CEM organism by the normal flora of the reproductive tract. The Veterinary Record. 133 (6), 125.

[24] Swerczek, T. W. (1979) Aggravation of Strangles, Equine Clostridial Typhlocolitis (Colitis X) and Bacterial Venereal Diseases in the Horse by Antibacterial Drugs. Proc AAEP, Miami Fl. pp 305-310.

[25] Richmond, R. (2015) Practical management of strangles outbreaks in horses. Aust Vet J. 93(8), N20.
[26] Mallicote, M. (2015) Update on Streptococcus equi sub sp equi infections. Vet Clin North Am Equine Pract. 31(1), 27-41.

[27] Waller, A. S. (2013) Strangles: taking steps towards eradication. Vet Microbiol. 167, 50-60. doi: 10.1016/j.vetmic.2013.03.033.

[28] Holland R. E., Harris, D. G., Monge, A. (2006) How to control strangles infections on the endemic farm. Proc 52nd Annu Meet AAEP. 78-80.

[29] Sweeney, C. R., Timoney, J. F., Newton, R., Hines, M. (2005) Control, and Prevention of Strangles Infections in Horses: Guidelines for Treatment. J Vet Intern Med. 19, 123-134. 